\title{
BIGENETIC PROPERTIES OF FINITELY GENERATED HYPER- (ABELIAN-BY-FINITE) GROUPS
}

\author{
Dedicated to the memory of Hanna Neumann
}

\author{
J. C. LENNOX
}

(Received 7 June 1972)

Communicated by M. F. Newman

\section{Introduction}

Let $\mathfrak{X}$ be a class and $\mathscr{P}$ a property of groups. We say that $\mathscr{P}$ is a bigenetic property of $\mathfrak{X}$-groups (or more simply, $\mathscr{P}$ is bigenetic in $\mathfrak{X}$-groups) if an $\mathfrak{X}$-group $G$ has the property $\mathscr{P}$ whenever all two-generator subgroups of $G$ have $\mathscr{P}$.

As an example we recall the well known fact that the property of being nilpotent is a bigenetic property both of the class of all finite groups and of the class of all finitely generated soluble groups. At one time it was hoped that nilpotency would turn out to be bigenetic in the class of all finitely generated groups or, failing that, at least in classes of finitely generated groups which in some sense were not too far from being finite or soluble. However Newman, using work of Golod and Šafarevič, constructed an example [11] of a three-generator infinite $p$-group ( $p$ a prime) with all of its two-generator subgroups nilpotent. His example was residually finite- $p$ so that nilpotency is not even bigenetic in the class of all finitely generated $p$-groups which are residually finite- $p$.

On the other hand it is not difficult to show, as we shall see, that nilpotency is bigenetic in the class of all finitely generated hyper-(Abelian-by-finite) groups. Our main object in this paper is to show further that a number of other properties are bigenetic in this class. Specifically we have as our principal result

THEOREM A. The properties of being polycyclic, nilpotent-by-finite, supersoluble, finite-by-nilpotent, nilpotent and finite are all bigenetic properties of the class of all finitely generated hyper-(Abelian-by-finite) groups.

We recall that if $\mathfrak{X}$ is a class of groups then a group is hyper- $\mathfrak{X}$ if it has an ascending invariant series each factor of which is an $\mathfrak{X}$-group.

We observe that Newman's example shows that none of the properties mentioned in Theorem A are bigenetic in the class of all finitely generated $p$-groups which are residually finite- $p$. 
Part of Theorem A provides a generalisation of the following known results about finite groups

LEMMA 1. Nilpotency, supersolubility and polycyclicity are bigenetic properties of the class of all finite groups.

If the two-generator subgroups of a group $G$ are nilpotent then $G$ is an Engel group. Since finite Engel groups are nilpotent, nilpotency is bigenetic in finite groups.

The fact that supersolubility is bigenetic in finite groups is a theorem of Carter, Fischer and Hawkes [3, Theorem 4.8] (for another proof see [18, Lemma 3.5]).

In order to see that polycyclicity is bigenetic in finite groups we recall that for finite groups polycyclicity and solubility are equivalent and we let $G$ be a counterexample to the assertion of least order. Then $G$ is a finite simple insoluble group with every proper subgroup and every two-generator subgroup soluble. Therefore $G$ is a minimal simple group and it follows from the work of Thompson [15] that $G$ is a two-generator group. Hence $G$ is soluble by hypothesis, a contradiction.

In [17] and [18] Wehrfritz has studied certain two-generator conditions in linear groups. For example in [17, Theorem 1] he proves that the property of being soluble-by-finite is bigenetic in the class of all subgroups of $G L(n, R)$, where $R$ is a finitely generated integral domain. However $I$ have been unable to decide whether the property of being soluble-by-finite is also bigenetic in finitely generated hyper-(Abelian-by-finite) groups.

Segal in [14] has shown independently and by a different method that the properties of being nilpotent-by-finite and supersoluble are bigenetic in the class of all finitely generated soluble groups. In addition he has considered twogenerator conditions in groups of automorphisms of certain classes of soluble groups.

\section{Proofs}

We denote the class of all finitely generated hyper-(Abelian-by-finite) groups by $\mathfrak{H}$. We first of all establish the fact that polycyclicity is bigenetic in the class of all $\mathfrak{S}$-groups (Theorem B). In order to do this we shall use the following argument which is due to Baer [2, \$2 Lemma 4] and which yields

LEMMA 2. If $\mathfrak{X}$ is a quotient closed class of groups such that finitely generated $\mathfrak{X}$-groups are finitely presented then in order to prove that a finitely generated group $G$ is an $\mathfrak{X}$-group it is sufficient to establish this fact in the case where every proper homomorphic image of $G$ is an $\mathfrak{X}$-group.

Proof. Suppose $G$ is a finitely generated group which is not an $\mathfrak{X}$-group. Let $N_{\lambda}(\lambda \in \Lambda)$ be a chain of normal subgroups of $G$ with the property that $G / N_{\lambda}$ is 
not in $\mathfrak{X}$ for all $\lambda \in \Lambda$. Let $N$ be the union of the $N_{\lambda}, \lambda \in \Lambda$. If $G / N$ is in $\mathfrak{X}$ then, since $G$ is finitely generated, $G / N$ is finitely presented and therefore $N$ is the normal closure in $G$ of finitely many elements $a_{1}, \cdots, a_{n}$ say, of $N$. Hence $N=N_{\lambda}$ for some $\lambda \in \Lambda$ which is impossible. Hence $G / N$ is not an $\mathfrak{X}$-group. We may now apply Zorn's Lemma to give the existence of a normal subgroup $H$ of $G$ maximal with respect to $G / H$ not in $\mathfrak{X}$. We may assume that $H=1$, and therefore that every proper homomorphic image of $G$ is in $\mathfrak{X}$. The proof is complete.

We now prove

THEOREM B. Polycyclicity is a bigenetic property of $\mathfrak{S}$-groups.

Proof. Suppose $G$ is an $\mathfrak{G}$-group such that every two-generator subgroup of $G$ is polycyclic. Since polycyclic groups are finitely presented (see [6, Lemma 1]) it follows by lemma 2 that we may assume that every proper homomorphic image of $G$ is polycyclic but that $G$ itself is not polycyclic.

Since $G$ is an $\mathfrak{H}$-group it is easy to see that there is a non-trivial normal subgroup $H$ of $G$ such that $H$ is finite or Abelian. If $H$ is finite it is polycyclic since, by lemma 1 , polycyclicity is bigenetic in finite groups. Moreover $G / H$ is polycyclic and therefore $G$ is itself polycyclic, a contradiction. Therefore $H$ is Abelian. Let $1 \neq a \in H$ and set $A=\left\langle a^{G}\right\rangle$, the normal closure of $a$ in $G$. Then $A$ is Abelian and $G / A$ is polycyclic by hypothesis. There is therefore a series

$$
A=G_{0} \triangleleft G_{1} \triangleleft \cdots \triangleleft G_{n-1} \triangleleft G_{n}=G
$$

of finite length such that $G_{i} / G_{i-1}$ is cyclic for $i=1, \cdots, n$. We may therefore set $G_{i}=\left\langle G_{i-1}, g_{i}\right\rangle$ for some $g_{i} \in G_{i}, i=1, \cdots, n$. Let $B_{r}=\left\langle a^{G_{r}}\right\rangle$. We prove by induction on $r$ that $B_{r}$ is finitely generated.

Now $B_{1}=\left\langle a^{\left\langle g_{1}\right\rangle}\right\rangle \leqq\left\langle a, g_{1}\right\rangle$, clearly, and $\left\langle a, g_{1}\right\rangle$ is polycyclic by hypothesis. Hence $\left\langle a, g_{1}\right\rangle$ satisfies the maximal condition on subgroups and so $B_{1}$ is finitely generated. Suppose $B_{r}$ is finitely generated for some $r \geqq 1$. Say $B_{r}=\left\langle b_{1}, \cdots, b_{s}\right\rangle$. Since $G_{r} \triangleleft G_{r+1}$ it follows that $B_{r+1}=B_{r}^{\left\langle g_{r+1}\right\rangle}$. Whence

$$
B_{r+1}=\left\langle b_{1}^{\left\langle g_{r+1}\right\rangle}, \cdots, b_{s}^{\left\langle g_{r+1}\right\rangle}\right\rangle
$$

Moreover each $\left\langle b_{j}^{\left\langle g_{r+1}\right\rangle}\right\rangle$ is finitely generated since it is a subgroup of the polycyclic group $\left\langle b_{j}, g_{r+1}\right\rangle j=1, \cdots, s$. Hence $B_{r+1}$ is finitely generated. By induction $B_{r}$ is finitely generated for $r=1, \cdots, n$ and so $A\left(=B_{n}\right)$ is finitely generated. However finitely generated Abelian groups are polycyclic and hence $G$ is polycyclic, a contradiction. The proof of Theorem B is now complete.

COROLlaRY B1. The property of being nilpotent-by-finite is bigenetic in $\mathfrak{H}$-groups.

Proof. Suppose $G$ is an $\mathfrak{5}$-group such that every two-generator subgroup of $G$ is nilpotent-by-finite but $G$ is not nilpotent-by-finite. Since finitely generated nilpotent- 
by-finite groups' are finitely presented it follows from lemma 2 that we may assume that every proper homomorphic image of $G$ is nilpotent-by-finite. Since $G$ is in $\mathfrak{H}$ there is a non-trivial normal subgroup $H$ of $G$ which is either Abelian or finite. By hyp thesis $G / H$ is nilpotent-by-finite. By coming down to a subgroup of finite index in $G$ if necessary, we may assume that $G / H$ is nilpotent. If $H$ is finite then $G$ is finite-by-nilpotent and it follows by a result of Hall [7, Theorem 2] that $G$ is nilpotent-by-finite, a contradiction. Therefore $H$ is Abelian and so $G$ is Abelian-by-nilpotent and therefore soluble. Since every two-generator subgroup of $G$ is nilpotent-by-finite, it is polycyclic and so $G$ is polycyclic by Theorem B. The final contradiction which completes the proof of Corollary B1 now follows from

LEMMA 3. The property of being nilpotent-by-finite is bigenetic in polycyclic groups.

Proof. Suppose $G$ is a polycyclic group with each of its two-generator subgroups nilpotent-by-finite. By induction on the length of a cyclic series for $G$ we may assume that $G=\langle H, x\rangle$, where $H$ is a nilpotent-by-finite normal subgroup of $G$ and $G / H$ is infinite cyclic. It follows that there exists a nilpotent characteristic subgroup $K$ of finite index in $H$. Thus $G / K$ is finite-by-infinite-cyclic and therefore is also infinite-cyclic-by-finite. Hence by coming down to a subgroup of finite index in $G$ we may assume that $G / K$ is infinite cyclic and so $G=\langle K, y\rangle$ for some $y$ in $G$. We may also assume that every proper homomorphic image of $G$ is nilpotent-by-finite.

Let $N=\zeta_{1}(K)$, the centre of $K$. Since $G$ is polycyclic $A$ is finitely generated, say $A=\left\langle a_{1}, \cdots, a_{n}\right\rangle$. By hypothesis the subgroups $\left\langle a_{i}, y\right\rangle$ are nilpotent-by-finite for $i=1, \cdots, n$. It follows that $\left\langle a_{1}^{m}, \cdots, a_{n}^{m}, y^{m}\right\rangle$ is nilpotent for some positive integer $m$. In other words $L=\left\langle A^{m}, y^{m}\right\rangle$ is nilpotent. If $A^{m}=1$ then $A$ is finite and therefore $K$ is finite, since a finitely generated nilpotent group with finite centre is finite by $[8$, Lemma 7$]$. Hence $G$ is nilpotent-by-finite as required. So we may assume that $A^{m} \neq 1$. It follows that $A^{m} \cap \zeta_{1}(L) \neq 1$. Set $M=\left\langle K, y^{m}\right\rangle$. Then we clearly have $B=\zeta_{1}(M) \neq 1$. Now $B \triangleleft G$ and $G / B$ is nilpotent-by-finite. Hence $M$ is nilpotent-by-finite and therefore $G$ is nilpotent-by-finite since $M$ is of finite index in $G$.

Remarks. (i) It is not difficult to deduce from Corollary B1 that the property of being Abelian-by-finite is also bigenetic in $\mathfrak{H}$-groups.

(ii) It is possible to prove Corollary B1 directly and then to deduce Theorem B from it. The proof proceeds by first reducing via lemma 2 to the case where the group $G$ in question is a finitely generated Abelian-by-nilpotent group with each of its two-generator subgroups nilpotent-by-finite. The fact that in this case $G$ is nilpotent-by-finite may be deduced from a parallel result for Noetherian modules for finitely generated nilpotent groups, namely: if $\Gamma$ is a finitely generated nilpotent 
group and $A$ is a Noetherian $\Gamma$-module with the property that the subgroup $\langle a, x\rangle$ of the split extension $A \cdot \Gamma$ is nilpotent-by-finite for all $a \in A, x \in \Gamma$ then there is a subgroup $\Delta$ of finite index in $\Gamma$ which acts unipotently on $A$. This result may be proved using the methods developed in [9] and [10].

\section{COROLlary B2. Supersolubility is bigenetic in $\mathfrak{H}$-groups.}

Proof. Suppose $G$ is an $\mathfrak{H}$-group such that every two-generator subgroup of $G$ is supersoluble. Since supersoluble groups are polycyclic it follows from Theorem $B$ that $G$ is polycylic. Corollary B2 now follows from

\section{Lemma 4. Supersolubility is bigenetic in polycyclic groups.}

Proof. Suppose false and let $G$ be a counterexample having a cyclic series of least length. Since supersolubility is bigenetic in finite groups $G$ is infinite. Also since $G$ satisfies the maximal condition on subgroups we may assume that every proper homomorphic image of $G$ is supersoluble.

We may now either note that $G$ is a polycyclic group with every finite homomorphic image supersoluble and is therefore supersoluble by [18, Lemma 2.2] or we may proceed directly as follows.

It is clearly enough to show that $G$ has a cyclic normal subgroup in order to obtain a contradiction. It follows that we may assume that $G$ has trivial centre. If $G^{\prime}$, the derived subgroup of $G$, is finite then by [7, Theorem 2] some term of the upper central series of $G$ is of finite index in $G$. Since $G$ has trivial centre it follows that $G$ is finite, a contradiction. Hence $G^{\prime}$ is infinite.

If $L$ is a normal subgroup of finite index in $G^{\prime}$ then $K=\cap_{g \in G} L^{0}$ also has finite index in $G^{\prime}[13,7.1 .6]$. Hence $K \neq 1$, since $G^{\prime}$ is infinite, and so by hypothesis $G / K$ is supersoluble. Therefore $G^{\prime} / K$ is nilpotent and so in particular $G^{\prime} / L$ is nilpotent. It now follows from a result of Hirsch (see e.g. [13, 7.1.12]) that $G^{\prime}$ is nilpotent. Again since $G^{\prime}$ is infinite we have that $A=\zeta_{1}\left(G^{\prime}\right)$ is nontrivial. Now by the minimality hypothesis on the cyclic length of $G$ there exists a normal subgroup $H$ of $G$ such that $G / H$ is cyclic and $H$ is supersoluble. Then $G=\langle H, g\rangle$ for some $g \in G$ and clearly $G^{\prime} \leqq H$. Now $A \triangleleft G$ and since $H$ is supersoluble there exists $1 \neq \alpha^{\prime} \in A$ such that $\langle a\rangle \triangleleft H$.ISet $K=\langle a, g\rangle$. Then $K$ is supersoluble by hypothesis and it follows that there exists $1 \neq b \in\left\langle a^{K}\right\rangle$ such that $\langle b\rangle \triangleleft K$. We show that $\langle b\rangle \triangleleft H$ whence $\langle b\rangle$ is a cyclic normal subgroup of $G$.

We may write $b$ in the form

$$
b=\prod_{i=1}^{n}\left(a^{g_{i}}\right)^{s_{i}}
$$

$r_{i}, s_{i}$ integers, $i=1, \cdots, n$ for some $n$.

Let $h \in H$. Then we have 


$$
b^{h}=\prod_{t=1}^{n}\left(a^{g^{r} t h}\right)^{s_{t}}
$$

Now

$$
g^{r_{i}} h=h\left[h, g^{-r_{i}}\right] g^{r_{i}}
$$

so that since $a \in \zeta_{1}\left(G^{\prime}\right)$ we obtain

$$
a^{g^{r_{i} h}}=a^{h g^{r},} \quad i=1, \cdots, n
$$

Now $a^{h}=a^{m}$ for some integer $m$ since $\langle a\rangle<H$ and it follows at once that $b^{n}=b^{m}$. Therefore $\langle b\rangle \triangleleft H$ and the proof is complete.

COROLLARY B3. The property of being finite-by-nilpotent is bigenetic in S-groups.

Proof. Suppose $\mathbf{G}$ is an $\mathfrak{5}$-group such that every two-generator subgroup of $G$ is finite-by-nilpotent. By the result of Hall cited earlier [7, Theorem 2] together with Corollary B1 it follows that $G$ is nilpotent-by-finite. Hence there exists a nilpotent normal subgroup $K$ of $G$ with $G / K$ finite. Since $G$ is finitely generated so also is $K$ and therefore $G$ satisfies the maximal condition on subgroups. In particular $G$ satisfies the maximal condition on normal subgroups and we may therefore assume that $G$ is not finite-by-nilpotent but that every proper homomorphic image of $G$ is finite-by-nilpotent. Furthermore, since finitely generated nilpotent groups are torsion-free-by-finite (this is easy to see directly or see $[13$, 7.1.11]) we may assume that $K$ is torsion-free. Since $G$ is not finite-by-nilpotent $K$ is non-trivial and therefore its centre $A$ is non-trivial. Let $a \in A, x \in G$. By hypothesis $\langle a, x\rangle$ is finite-by-nilpotent and so the repeated commutator $[a, x, \cdots, x]$ is an element of $A$ of finite order for sufficiently many $x$ 's. But $A$ is torsion-free and so $x$ acts unipotently on $a$. Moreover $A$ is finitely generated and it follows easily that $x$ acts unipotently on $A$. We chose $x$ arbitrary in $G$ and since $K$ centralizes $A$ and $G / K$ is finite we have that $G$ acts nilpotently on $A$ (see e.g. [16, Lemma 8.1]). Therefore $A \leqq \zeta_{m}(G)$, the $m$-th term of the upper central series of $G$, for some $m \geqq 1$. By hypothesis $H=G / A$ is finite-by-nilpotent and Hall's result [7, Theorem 2] shows that $H / \zeta_{r}(H)$ is finite for some $r$. Therefore $G / \zeta_{m+r}(G)$ is finite since $A \leqq \zeta_{m}(G)$. From a theorem of Baer [1, page 369] it follows that $G$ is a finite-by-nilpotent group. This contradiction completes the proof.

REMARK. It is not difficult to deduce from Corollary B3 that the property of being finite-by-Abelian is bigenetic in $\mathfrak{H}$-groups.

We finish the proof of Theorem A with two short lemmas.

LEMMA 5. Nilpotency is bigenetic in $\mathfrak{5}$-groups.

Proof. Suppose $G$ is an $\mathfrak{S}$-group with every two-generator subgroup nilpotent. 
Then since nilpotency is bigenetic in finite groups it is clear that every finite image of $G$ is nilpotent. It follows by a result of Robinson [12] that $G$ is nilpotent.

Alternatively lemma 2 and the results of Gruenberg [4] and [5] on Engel groups may be used to obtain the same result.

LeMma 6. Finiteness is bigenetic in $\mathfrak{H}$-groups.

Proof. Suppose $G$ is an $\mathfrak{G}$-group such that every two-generator subgroup of $G$ is finite. Since finite groups are finitely presented it follows from lemma 2 that we may assume that all proper homomorphic images of $G$ are finite. It is then easy to deduce that $G$ must be finite.

\section{References}

[1] R. Baer, 'Representations of Groups as Quotient Groups II', Trans. Amer. Math. Soc. 58 (1945), 348-389.

[2] R. Baer, 'Nilgruppen', Math. Z. 62 (1955), 402-437.

[3] R. W. Carter, B. Fischer and T. Hawkes, 'Extreme Classes of Finite Soluble Groups', J. Algebra 9 (1968), 285-313.

[4] K. W. Gruenberg, 'Two Theorems on Engel Groups', Proc. Cambridge Philos. Soc. 49 (1953), 377-380.

[5] K. W. Gruenberg, 'The Engel Elements of a Soluble Groups', Illinois J. Math. 3 (1959), 151-168.

[6] P. Hall, 'Finiteness Conditions for Soluble Groups', Proc. London Math. Soc. (3) 4 (1954), 419-436.

[7] P. Hall, 'Finite-by-nilpotent groups', Proc. Cambridge Philos. Soc. 52 (1956), 611-616.

[8] P. Hall, 'On the Finiteness of Certain Soluble Groups', Proc. London Math. Soc. (3) 9 (1959), 595-622.

[9] J. C. Lennox and J. E. Roseblade, 'Centrality in Finitely Generated Soluble Groups', J. Algebra 16 (1970), 399-435.

[10] J. C. Lennox, 'A Note on a Centrality Property in Finitely Generated Soluble Groups', to appear in Proc. Cambridge Philos. Soc.

[11] M. F. Newman, 'A Theorem of Golod-Šafarevič and an application in group theory', unpublished.

[12] D. J. S. Robinson, 'A Theorem on Finitely Generated Hyperabelian Groups', Invent. Math. 10 (1970), 38-43.

[13] W. R. Scott, Group Theory, (Prentice Hall, Englewood Cliffs, N.J. 1964).

[14] D. Segal, 'Groups of Automorphisms of Infinite Soluble Groups', Proc. London Math. Soc. (3) 26 (1973) 630-652.

[15] J. G. Thompson, 'Non solvable finite groups all of whose local subgroups are solvable', Bull. Amer. Math. Soc. 74 (1968), 383-437.

[16] B. A. F. Wehrfritz, Infinite Linear Groups, (Queen Mary College Mathematics Notes, London, 1969.)

[17] B. A. F. Wehrfritz, '2-generator conditions in linear groups', Arch. Math. (Basel) 22 (1971), 237-241.

[18] B. A. F. Wehrfritz, 'Supersoluble and Locally Supersoluble Linear Groups', J. Algebra 17 (1971), 41-58.

University College Cardiff, CFI IXL

Wales 